\title{
Participación política de las mujeres en Chile y sus oportunidades, en la percepción de estudiantes de primer año de universidad. El caso de la Universidad de Concepción ${ }^{1}$
}

Political Participation and opportunities for women in Chile, as perceived by first-year students at the University of Concepción

\author{
Ximena GAUCHÉ MARCHETTI ${ }^{2}$, CARLOS MUÑOZ LABRAÑA ${ }^{3}$, \\ CECILIA BUSTOS IBARRA ${ }^{4}$ Y AMAYA ÁLVEZ MARÍN 5 \\ Universidad de Concepción, Chile
}

RECEPCIÓN: I 8/O7/20I6• ACEPTACIÓN: 3 I/o8/20I 6

I. Este artículo forma parte de la investigación enmarcada en el Proyecto «Los conocimientos y actitudes respecto a la democracia y la ciudadanía que poseen los estudiantes que ingresan a la Universidad de Concepción», Proyecto de Investigación Asociativo financiado por la Vicerrectoría de Investigación y Desarrollo (VRID) de la Universidad de Concepción, Código 2I2I6I007-I.o (20I2-20I5).

2. Abogada. Doctora en Derecho, Universidad Autónoma de Madrid e Instituto Universitario de Investigación Ortega y Gasset, España. Académica de la Facultad de Ciencias Jurídicas y Sociales de la Universidad de Concepción, Chile. Correo: xgauche@udec.cl

3. Doctor en Educación, Universidad de Concepción, Chile. Académico de la Facultad de Educación de la Universidad de Concepción, Chile. Correo: carlosem@udec.cl

4. Candidata a Doctora en Sociología, Universidad Autónoma de Barcelona. Académica del Departamento de Trabajo Social de la Facultad de Ciencias Sociales, Universidad de Concepción, Chile. Correo: cecbustos@udec.cl

5. Abogada. Doctora en Derecho, Universidad de York, Canadá. Académica de la Facultad de Ciencias Jurídicas y Sociales de la Universidad de Concepción, Chile. Correo: aalvez@udec.cl 
RESUMEN Esta investigación da cuenta de algunas concepciones y actitudes que posee el estudiantado de carreras de las áreas de educación, ciencias sociales y ciencias jurídicas de la Universidad de Concepción, sobre algunos aspectos vinculados a la participación política de las mujeres en Chile. Ello se ha obtenido a partir de los resultados de una encuesta aplicada a estudiantes de primer año. Tales resultados demostraron que al iniciar sus estudios universitarios valoran positivamente la consagración formal de la participación política de las mujeres, consideran un deber del Estado garantizar la igualdad de oportunidades y manifiestan desconfianza en los partidos políticos y en el Congreso Nacional. Sin embargo, a la hora de las oportunidades, la confianza tiende a ser mayor en los hombres para depositar responsabilidades de carácter político, lo que introduce la necesidad de avanzar desde la formalidad del buen discurso sobre igualdad a la acción práctica en política.

PALABRAS CLAVE Mujeres, política, participación, oportunidades.

ABSTRACT This research gives an account of several views and attitudes held by students in the areas of Education, Social Sciences, and Legal Sciences at the University of Concepción, related to the participation of women in politics in Chile. The results obtained from a survey given to first year students show that at the beginning of their university studies, students value the participation of women in politics, believe the State has a duty to ensure equal opportunities, and express distrust in political parties as well as the Chilean National Congress. However, in practice, men are more often entrusted with political responsibilities, highlighting the need to move away from a formal discourse of equality and towards practical action in politics.

KEYWORDS women, politics, participation, opportunities.

\section{introducción}

En I977, la Asamblea General de las Naciones Unidas proclamó el 8 de marzo como el «día internacional por los derechos de la mujer y la paz internacional». En 20I 5 esta conmemoración se enmarcó en un contexto internacional particular: se cumplieron 20 años desde la histórica Conferencia de Beijing sobre la Mujer que marcó un antes y un después al introducir la perspectiva de género al análisis de las demandas de derechos humanos de las mujeres. Por 
otro lado, se cumplió el plazo formal fijado para revisar el cumplimiento de los llamados «Objetivos de Desarrollo del Milenio» centrados en buena medida en lograr la plena igualdad entre hombres y mujeres en el mundo y en programar una nueva agenda centrada ahora en la sostenibilidad, proyectada para los próximos 30 años a través de los llamados ahora «Objetivos de Desarrollo Sostenible».

Por ello entonces, las Naciones Unidas ha decidido utilizar el lema «Empoderando a las mujeres. Empoderando a la humanidad. ¡Imagínalo!» con el que se busca recrear un mundo en que cada mujer y cada niña pueda elegir sus decisiones, sea en el ámbito de la política o la educación, y tener sus propias vivencias en sociedades libres de discriminación y de violencia ${ }^{6}$.

Para un país que tiene por segunda vez en su historia una Presidenta de la República, la fecha y el lema no pueden pasar desapercibidos. Las mujeres son - cifras más o cifras menos según el Censo que se quiera analizar - la mitad de la población de este país y, pese a ello, aún faltan muchos espacios en que ser protagonistas y gozar de ese empoderamiento que promueve la ONU.

Según la Décima Encuesta Nacional «Percepciones de las Mujeres sobre su situación y condiciones de vida en Chile 20I4", elaborada por la Corporación Humanas y presentada a la ciudadanía en diciembre $2014^{7}$, un $83,8 \%$

6. Recuperado el 22 de octubre de 2015, desde http://www.un.org/es/events/womensday/.

7. A partir del año 2004, Corporación Humanas realiza encuestas nacionales de opinión pública dirigidas a mujeres con el objetivo de: generar conocimiento en torno a las percepciones y demandas de las mujeres, supliendo el vacío de estudios de opinión tradicionales; visibilizar la percepción de las mujeres en temáticas no abordadas por los estudios de opinión existentes; e identificar permanencias y cambios en el discurso de las mujeres, produciendo información comparable en el tiempo. La Décima encuesta se aplicó entre el 22 de Septiembre al 27 de Octubre 20I4; el universo de estudio fueron mujeres mayores de i 8 años. En cuanto a la y la cobertura geográfica, la muestra utilizada representa la opinión de todas las mujeres pertenecientes por definición al Universo de Estudio, y que habitan en 3 I 3 Comunas de Chile, incluyendo todos los Distritos urbanos y casi todos los Distritos rurales de dichas Comunas. En este marco muestral habita más del 98,3\% de la población del país. Como tamaño muestral, hay que indicar que se entrevistó a I 235 mujeres, con un error muestral máximo de +/-3, I considerando un nivel de confianza de $95 \%$. El diseño muestral fue muestreo Interpenetrante de Mahalanobis, de áreas, trietápico, estrictamente probabilístico, sin reemplazos. Recuperado el I 2 de diciembre de 20I4, desde http://www.humanas.cl/?p=I40I 7 
de las mujeres encuestadas manifestó sentirse discriminada. De ese porcentaje, 7I,9\% consideró el ámbito político como uno en que se produce esta exclusión y el $53 \%$ estimó que Chile es un país machista. Específicamente en el ámbito de la participación política, la misma encuesta muestra que el 50,5\% cree que la política es sólo para hombres.

Si por otra parte consideramos que en los actuales cargos públicos de elección popular hay menos mujeres que hombres al igual que en los cargos de designación por confianza, parece importante seguir poniendo el tema de la participación política de las mujeres en el eje de los trabajos académicos y de investigación ligados a las mujeres y sus derechos, o a la democracia y la participación política.

En efecto, al final de 20 I 5 Chile podía dar cuenta de 6 senadoras de un universo de 38 integrantes de la Cámara Alta del Congreso Nacional; I 8 diputadas en una Cámara de Diputados que cuenta con I 20 integrantes; poco más de 50 alcaldesas en un país con más de 300 Municipios $^{8}$ y en todos los gobiernos desde I 990 a la fecha nunca el porcentaje de intendentas ha superado el $28 \%$ del total (Informe Auditoría a la Democracia, 20I0). Si bien la mirada positiva muestra avances interesantes en este ámbito - $y$ legislativamente se vienen tiempos más auspiciosos con la introducción de cuotas en el sistema electoral chileno a partir de la Ley 20.840 aprobada en 20 I 5 - algunos de estos avances tienen más bien un carácter simbólico que real en relación a su impacto futuro y al aseguramiento de mayores oportunidades de participación de las mujeres.

Con este contexto fáctico, más de iooo estudiantes de primer año de diferentes carreras de la Universidad de Concepción participaron voluntariamente durante $20 \mathrm{I} 3$ en una encuesta para entregar sus opiniones relativas a temas de ciudadanía, en I 2 dimensiones ${ }^{9}$ : democracia, buenos ciudadanos, estado, confianza en las instituciones, oportunidades, el sistema político, el currículo escolar, la acción política ( $\mathrm{I}$ y 2), la efectividad de la acción política, el uso de la fuerza militar y la atención a los medios de comunicación, cada una de las cuales presenta afirmaciones con las cuales el o la encuestado tuvo que identificarse en cuatro categorías en formato de Escala de Lickert, más una

8. Recuperado el I 2 de diciembre de 20I4, desde http://www.munitel.cl/.

9. Se trata de una investigación de carácter cuantitativa, que utilizó como instrumento para recoger la información la Encuesta Internacional de Educación Cívica diseñada por la International Association for the Evaluation of Educational Achievement. Más información sobre la encuesta, en el apartado sobre Metodología. 
categoría que se interpreta como «no sabe». Algunas de estas dimensiones correspondieron a preguntas generales relativas a ciudadanía, mientras que otras se relacionaron con la visión del o la estudiante sobre su experiencia particular o sobre la realidad que le rodea. Lo que se ha buscado es tener un diagnóstico respecto a los conocimientos, y actitudes sobre la democracia y la ciudadanía que poseen los y las estudiantes que ingresan a la Universidad, especialmente lo que consideran bueno y malo para la democracia, sus percepciones de lo que es un buen ciudadano y las responsabilidades que corresponde al Estado en la sociedad, su confianza en algunas de las instituciones más importantes del país, la regularidad con que discute acerca de la política nacional e internacional, así como lo que espera hacer una vez obtenido su título profesional en éstas materias, proporcionando elementos de juicio para que esta importante institución de educación superior, pueda adoptar las medidas que considere necesarias, para promover y dar continuidad a esta su formación.

Parte de las preguntas tuvo que ver con la percepción de los estudiantes desde distintos ámbitos que se relacionan con la participación política de las mujeres - sobre las oportunidades de ellas en política y la valoración de esta. Lo que pretende este artículo es exponer los resultados de la aplicación de esta encuesta en ese ámbito específico, a la luz del marco teórico que se expondrá previamente, que pone el énfasis en el género como categoría para analizar determinados fenómenos sociales y teniendo en vista para el análisis el contexto educativo actual sobre participación, entendiendo así que este artículo entonces puede ser una contribución principalmente a las disciplinas del derecho, la ciencia política y la educación.

A partir de ello, se quiere mostrar específicamente la percepción de la importancia de la participación de las mujeres en política en Chile, identificada a partir del análisis de preguntas concretas de la encuesta relacionada, específicamente en las carreras pertenecientes a las Facultades de Educación, de Ciencias Sociales, y de Ciencias Jurídicas y Sociales por la formación que reciben sus estudiantes, lo cual debe ser precisado pues los resultados deben ser tenidos en vista con la limitación del perfil estudiantil escogido.

A modo de precisión preliminar, se deja establecido que se ha asumido como premisa de esta investigación que definir de manera unívoca lo que hoy podemos entender por «sistema democrático» no es una tarea fácil y escapa con mucho a los objetivos de este proyecto. Sólo a fin de dotar de un contenido al término para efectos de este artículo, diremos que estamos entendiendo por tal, aquellos sistemas políticos referidos a la forma de organización del estado 
en que las decisiones son tomadas por los integrantes del cuerpo social, sea de manera directa ( «democracia directa»), sea de manera indirecta o representativa («democracia representativa»), o sea de forma participativa («democracia participativa»). Ahora bien, conforme la literatura consultada puede decirse que parece establecido el consenso en torno a que la participación política es un elemento común a cualquiera de las formas de democracia que se reconocen hoy en el mundo. Sobre el concepto de participación política, desde la Ciencia Política se puede encontrar una definición que dota de contenido a esta idea. Dice Gianfranco Pasquino: «La participación política es aquél conjunto de actos y de actitudes dirigidas a influir de manera más o menos directa y más o menos legal sobre las decisiones de los detentadores del poder en el sistema político o en cada una de las organizaciones políticas, así como en su misma selección, con vista a conservar o modificar la estructura (y por tanto los valores) del sistema de intereses dominantes». ${ }^{10}$ Dicha comprensión teórica será la que oriente este trabajo.

\section{Marco teórico}

Parece un hecho incuestionable que los derechos de las mujeres son parte integral del discurso actual, hablado en clave de igualdad, desarrollo y género. Ello responde al lugar secundario que se ha dado tradicionalmente a las mujeres en la mayoría de las sociedades. En efecto, considerando que en buena parte de las sociedades estatales existen sistemas de división y estratificación social, que sitúan a unas determinadas categorías de personas por encima de otras y que determinan situaciones diferentes de prestigio, poder y riqueza, las mujeres son manifestación clara de tales divisiones y estratificación social (Macionis \& Plummer, 2007) y aunque durante largo tiempo buena parte de los estudios desde las ciencias sociales se ocuparon preferentemente del sistema de estratificación y jerarquía social en función de la posición económica, el poder y el prestigio de las personas -incluyéndose en la posición social y económica por cierto las diferencias de clase- en tiempos más recientes se reconoce que además de las diferencias económicas entre las personas existen también otras diferencias importantes, como las que se refieren al género «que sitúan a los miembros de una sociedad en situaciones radicalmente diferentes en cuanto a sus expectativas o posibilidades de elegir y llevar adelante sus

io. PASQuino (I994) p. 2 Io. 
sueños y aspiraciones». ${ }^{11}$ Estas diferencias hacen ilusorio en muchos casos el paradigma moderno de la igualdad que mueve a la sociedad especialmente desde los tiempos de las revoluciones que vieron nacer a los derechos humanos como conceptos jurídicos.

En esa lógica de ideas es posible ver que las personas integramos diversas jerarquías que pueden presentarse como desigualdades sociales y económicas (en las que influyen el trabajo de una persona, su riqueza y sus ingresos); como desigualdades étnicas y de raza (en que influyen la raza y etnia de una persona); como desigualdades por razones etarias (en que influye la edad de una persona); como otros tipos de desigualdades (en que tienen influencia la discapacidad, el lenguaje y la nacionalidad de una persona, como factores creadores de la desigualdad, por ejemplo) y finalmente como desigualdades de género (en que influyen la posición de una persona como hombre o mujer) quedando en este último caso expuestas además a padecer los otros tipos de desigualdades que agravan la situación: mujeres pobres, mujeres indígenas, mujeres niñas y mujeres adultas mayores, mujeres migrantes. Todas estas diferencias suponen distintas manifestaciones en la vida social. ${ }^{12}$

Con estas premisas para mirar la realidad, es posible comprobar que las mujeres han sufrido por años todas estas manifestaciones de desigualdad en función primeramente de su pertenencia a este sexo y luego por la forma de construcción de las instituciones sociales, políticas y jurídicas que se ha hecho sobre la idea de abstracción respecto del sujeto que va a ser su destinatario, tal y como si se tratara de un ser "asexuado" con la bandera de la «neutralidad y abstracción» como requisito que debe tener una norma o una política pública. Ejemplos para confirmar esta afirmación surgen rápidamente al mirar la sociedad y su recorrido histórico. De muestra dos problemáticas: pobreza y violencia.

La llamada feminización de la pobreza es una constante desde hace años. Se manifiesta en estos tiempos, por ejemplo, a partir de la proliferación de hogares a cargo de madres solas que ganan un bajo salario o que son víctimas de explotación por sus jefes o de violencia doméstica de parte sus parejas, quedando así excluidas de participar activamente en la vida social. Mirada desde otro ángulo, esta cara femenina de la pobreza se refleja en que son las mujeres quienes reciben el mayor impacto de las consecuencias negativas de fenómenos

I I. Macionis y Plummer (2007) p.2 I 6

I2. Macionis y Plummer (2007) p. 2 I 6 
como la globalización y los ajustes económicos que trae (a partir de ser las primeras a quienes se les restringe el acceso y la continuidad laboral con la excusa de la maternidad); de todas las formas de comercio ilícito internacional (a partir de ser «ellas» y no «ellos» los principales «objetos» traficados en muchos países al modo casi de validar que se pueda hablar de ellas como un «objeto" anulando la condición de «sujeto» ${ }^{13}$ ); del mal uso de nuevas tecnologías (a partir de ser las principales víctimas por ejemplo de delitos de prostitución o pornografía en la red ${ }^{14}$ ) o de los conflictos internos o internacionales (a partir de que la satisfacción de las necesidades familiares básicas se ven mermadas antes que cualquier intento de reducir armamentos siendo mujeres generalmente las principales gestoras de estas necesidades en muchos contextos sociales y considerando que junto a los niños y niñas son las principales víctimas de los conflictos armados ${ }^{15}$ ).

También la violencia a mujeres, usualmente «de género» y no sólo «intrafamiliar» o "doméstica», es una patente y viva manifestación de las desigualdades que afectan a mujeres. El tema ha tomado forma en normas internaciona-

I3. Según la Oficina de las Naciones Unidas contra el Delito (UNODC por su sigla en español) las cifras de la Organización Internacional del Trabajo (OIT), indican que cerca de 2,4 millones de personas en el mundo son víctimas de trata de personas y son sometidas a trabajos forzosos. De las víctimas identificadas, el $80 \%$ son mujeres y niñas. Informe «La trata de personas: la cruda realidad». Recuperado el 20 de enero de 20I 5, desde http://www.unodc.org/documents/blueheart/factsheet_spanish.pdf

I4. En el año 2000 la UNESCO creó el Programa «Información para Todos», conocido por sus siglas en inglés IFAP (Information for All Programme), el cual se sustenta mediante el aporte de algunos países donantes y uno de cuyos objetivos es trabajar por las implicancias éticas, sociales y legales del acceso a las nuevas tecnologías. En esa línea, junto con fomentar el acceso igualitario para todas y todos para aprovechar los beneficios de ellas, en el marco de los derechos básicos de libertad de expresión, acceso a la información, derecho a la educación y derecho a participar en la vida cultural, se busca tomar medidas para evitar los abusos y proteger a la población de los riesgos, entre los cuales tiene lugar especial garantizar la seguridad de las niñas en internet por este mal uso. Recuperado el 20 de enero de 20 I $_{5}$, desde http://portal.unesco.org/ci/en/ ev.php-URL_ID=I627\&URL_DO=DO_TOPIC\&URL_SECTION=20. html

I 5 . Así lo ha reconocido el Consejo de Seguridad en el Preámbulo de la Resolución I325, de 2000 y en la que se entiende su principal continuación, la Resolución I820, de 2008 . Otras resoluciones en similar sentido son las número i 888 y I 889 , ambas de 2009; I960, del año 20I0; y 2 I06 y 2122, ambas del año 20I3. 
les, con el texto emblemático de la Convención Interamericana para prevenir, sancionar y erradicar la violencia contra la mujer, también conocida como Convención de Belém do Pará16, y es recurrente en foros sociales y políticos internacionales y nacionales, constatándose cada vez de forma más abierta que se trata de un problema que traspasa las barreras de lo socio cultural y de las diferencias entre países.

Así entonces, la cuestión de la estratificación social hoy tiene en la cuestión del género una categoría social que ayuda a determinar cómo se dan las relaciones humanas en una sociedad. Al igual que otras categorías como la raza, la lengua o la etnia, está claro que incide y genera exclusión en diferentes ámbitos, entre ellos el político.

Todo lo anterior por cierto ha sido desde la constatación de que la cultura «humana» ha tenido y tiene una fuerte carga androcéntrica en la construcción e implementación de la mayoría de sus instituciones, desde las políticas y sociales a las jurídicas, estructurando espacios y lugares sólo para hombres (lo público) y espacios y lugares sólo para mujeres (lo privado) y reflejándose en el lenguaje usado por esas instituciones. Expresiones como «los hombres», «los individuos», «las personas», «los ciudadanos» se han venido haciendo sinónimos de hombres y mujeres y hemos tenido que entender por siglos que quedamos incorporadas en esas categorías que toman lo masculino como lo universal en el marco de una categoría bifrontal que aspira a una mal entendida neutralidad que, como ha dicho alguna autora nacional, le ha pasado una mala jugada a las mujeres.

Dicho en otras palabras, el mundo y sus avances se han mirado tradicionalmente con ojos masculinos a partir de una sociedad sexista y patriarcal que, aunque asume diversas formas en los distintos contextos espacio-temporales se estructura en lo general en esa línea de una relegación o hasta olvido de la existencia de las mujeres y sus circunstancias. Parafraseando palabras de mujer bien se puede decir que la mitad de la población — las mujeres - han estado silenciadas. ${ }^{17}$ Sin voz para poder interpretar la realidad o poder modificarla. La humanidad se ha construido sobre su silencio y sobre su sumisión la cultura patriarcal ha esculpido una imagen a su medida que se supone es para toda la

16. Adoptada el 9 de junio de 1994 en Brasil y en vigor internacional desde el 5 de marzo de 1995. Esta convención fue firmada por Chile en 1995, entrando en vigor para el país el año I996.

I7. Renau (2008) p. I3. 
humanidad pero responde en verdad a un humano varón que se construye y sigue construyéndose a sí mismo. «Un arquetipo que ha ido variando a lo largo de la historia, pero que mantiene hasta hoy sus potentes rasgos esenciales» (Renau, 2008).

En este orden de ideas es que se ha desarrollado el sexismo, como «la creencia de que el sexo propio es, por definición, superior», convirtiéndose en un elemento importante que contribuye a sostener este patriarcado, entendiendo el patriarcado (literalmente «el dominio del padre») como una forma de organización social por la que los hombres dominan, oprimen y explotan a las mujeres. ${ }^{18}$ Podría decirse que en lenguaje coloquial el sexismo es lo que se llama habitualmente «machismo» —al que refiere la encuesta de la Corporación «Humanas» referida en la introducción - y genera en la práctica múltiples episodios de discriminación o de lo que se conoce también como misoginia, es decir y en términos simples, una conducta hostil y hasta odiosa hacia las mujeres.

A estas ideas habría que agregar que como soporte de este sistema de dominación —aun cuando la idea de rol de género no estaba siquiera concebida teóricamente- ayudó la disociación de una serie de aspectos de la vida humana, a partir de los cuales todos aquellos que supuestamente no generaban mayor aporte al desarrollo social (o aporte materialmente visible) como los afectos y sus manifestaciones físicas, las cuestiones domésticas del hogar común o el hecho biológico de la maternidad, han sido atribuidos a las mujeres con una consiguiente carga valórica de inferioridad. Así, la racionalidad parece ser atributo de hombres y la emocionalidad de mujeres. Afirmación que hoy es insostenible y cae por su propio peso con la fuerza de la evidencia que entrega la realidad.

En este marco, así como en un momento el socialismo entre otras razones nace para luchar contra el sistema capitalista o de clases - en una acepción marxista pura de la idea de capitalismo- el feminismo se alza en pleno siglo XVIII como una doctrina que busca luchar contra el sexismo y contra el patriarcado como sistema de dominación de un sexo sobre otro y en virtud del cual y como recuerda Dolores Renau (Renau, 2008), las mujeres han estado ausentes de los tratados que nos explican el devenir de la humanidad, la construcción de las leyes que ordenan la vida colectiva o los más altos productos del espíritu humano. ${ }^{19}$

i 8. Macionis y Plummer (2007) p. 338

I9. Beltrán et al. (2010) p.27. 
Así, las primeras teorías feministas nacen para luchar contra el patriarcado que, en cuanto ordenación social y política dejaba a las mujeres en un segundo plano en todas las decisiones de lo público. Encuentra su raíz en las Revoluciones francesa e inglesa que en su visión particular cada una propugnaba la «igualdad» para «todos». De esta época datan dos obras claves para el análisis de la materia: «Una reivindicación de los derechos de la mujer», escrita en I792 por Mary Wollstonecraft y «El sometimiento de la mujer» (obra clásica del liberalismo) escrita en I 869 por John Stuart Mill y su esposa Harriet Taylor Mill, quien influyó para que el inglés incorporara los derechos de la mujer en su análisis, considerando que las desigualdades entre los sexos son un mal en sí mismas y un obstáculo para el progreso de la humanidad, rechazando las justificaciones biologicistas sobre la supuesta condición inferior de las mujeres. Antes en todo caso, la obra "Sobre la igualdad de los sexos", de Poulain de la Barre, escrita en I673, había puesto en el debate el tema de la demanda por igualdad sexual. ${ }^{20}$

En estas primeras teorías feministas, llamadas modernas 21 , lo que se buscaba eran mejoras educativas e igualdad de las mujeres ante la ley, como la obtención del derecho a voto que por ejemplo las mujeres británicas ejercieron por primera vez recién en las elecciones generales del I4 de diciembre de I9I 8 en lo que se ha llamado por los historiadores el «movimiento de las sufragistas ${ }^{22}$ y como puede apreciarse por el momento histórico en que nacen lo hacen inmersas en los planteamientos de la modernidad sobre ciudadanía e igualdad, en medio de la Ilustración y las vindicaciones por individualidad, autonomía y derechos, de cara a la participación política entre otras demandas.

Así, el inicio fuerte de las reivindicaciones femeninas en este tiempo contemporáneo tuvo como escenario la época de los reclamos sociales; no sólo sexistas sino también raciales, pacifistas y estudiantiles de la segunda mitad del siglo XX y una clara impronta vinculada a la participación política. Todos ellos fortalecieron la causa de las mujeres por dejar de ser consideradas

20. Fries y Matus (i999) p. I9

2i. Beltrán et al. (20IO) p. I 2.

22. En Estados Unidos, el movimiento sufragista estuvo inicialmente muy relacionado con el movimiento abolicionista y un gran número de mujeres unieron sus fuerzas para combatir en la lucha contra la esclavitud. En I 848, en el Estado de Nueva York, se aprobó la Declaración de Seneca Falls, uno de los textos fundacionales del sufragismo en este lado del planeta. 
un sexo secundario y fueron influyendo en mayor o menor grado para que se desarrollaran nuevas vertientes de entender las reivindicaciones, llegando hoy por ejemplo, al tema de la familia y la sexualidad.

En palabras de Renau, el feminismo es afortunadamente un extraordinario instrumento crítico colectivo, una filosofía global que se enriquece cada día con nuevas propuestas gracias a la radicalidad de sus planteamientos, a su arraigo vital y a la urgencia social que lo sustenta y que puede aumentar su aporte cuando toma la perspectiva de género, construcción social que lleva a entender que lo femenino (y también lo masculino) tiene distintas formas de manifestarse en los diversos contextos espacio-temporales y afecta la distribución del poder en la organización social respectiva. En ese sentido, la perspectiva de género supone considerar al género como una categoría de análisis que plantea «una multiplicidad de propuestas, programas y acciones alternativas a los problemas sociales que generan la desigualdad entre los géneros» (Uña y Hernández, 2004).

A esta altura viene bien recordar que no se pueden hacer sinónimos género y mujer. Hacerlo supone un reduccionismo que justamente priva de toda utilidad al desarrollo de la categoría género, no sólo para las propias mujeres sino también frente a las reivindicaciones de hombres que se amparan en su género para luchar contra ciertas discriminaciones, por ejemplo, por su orientación sexual, tema olvidado en las burocracias estatales e internacionales que tienden a asumir la implantación de las cuestiones de género a partir de desarrollos centrados en la mujer y concebir a los hombres como una categoría única y a lo masculino como una identidad sin matices.

Así las cosas, en lo expuesto encontramos en buena medida las bases en las cuales se insertan las razones de la menor participación política de las mujeres a lo largo del tiempo. En efecto, esta menor participación es también una constante en la historia y una manifestación de desigualdad hoy visible tanto como la pobreza femenina o la violencia a las mujeres. Ellas siguen infra representadas, sea en los procesos de elección, como votante o potencial elegible, o sea en el acceso a cargos público-políticos.

No obstante, hay que reconocer que esta es una situación que tiende al cambio favorable en las últimas décadas y prueba de ello es que al segundo semestre del año 2015 era posible dar cuenta de varios países que tienen o han tenido una mujer como Jefa de Estado y/o de Gobierno elegidas en procesos electorales democráticos ${ }^{23}$.

23. A 20 I 5 , los siguientes estados son ejemplo ya que están o han estado gobernados 
Por otro lado, varios son los países que han incorporado en sus sistemas políticos electorales el sistema de «cuotas» como una clase de las llamadas «acciones positivas» - $\mathrm{O}$ "medidas especiales temporales» para el derecho internacional de los derechos humanos-y las políticas de paridad, lo que se traduce en un paliativo al menos formal para la desigualdad en participación femenina en política.

En el caso de América Latina, continente que mantiene aún serios déficit en términos de participación política de mujeres ${ }^{24}$, se han ido produciendo reformas legislativas importantes que tienen que ver con la implementación de leyes electorales que obligan a los partidos políticos a incorporar principios y mecanismos de "discriminación positiva", como son las cuotas o cupos de representación, para garantizar la efectiva integración de mujeres en cargos electivos de decisión al interior de los partidos políticos y del Estado. Las cuotas de participación (voluntarias u obligatorias) buscan garantizar que las mujeres constituyan un porcentaje de al menos un $30 \%$ o $40 \%$ y tienen una aplicación temporal, con el límite que suponga el término de las barreras que dificultan el acceso de las mujeres a la política. Los cupos de representación, en tanto hacen referencia a la asignación efectiva de un mínimo de escaños reservados a las mujeres en las legislaturas nacionales, debiendo tener también un carácter transitorio. El primer país en incorporar cuota en su legislación fue Argentina en I99I, reconociendo un $30 \%$ de mujeres en sus listas electorales para postularse al poder legislativo. Con los años el grupo se fue ampliando y hacia el final de $20 \mathrm{I} 4$ se podían contar en varios los países de este grupo continental

por una mujer electa en procesos democráticos: Alemania (Angela Merkel); Argentina (Cristina Fernández); Chile (Michelle Bachelet); Costa Rica (Laura Chichilla); Filipinas (Gloria Macapagal); Finlandia (Tarja Halonen); Liberia (Ellen Johnson); Irlanda (Mary Mcallese); Islandia (Johanna Sigurdardóttir); Mozambique (Luisa Diogo). Además, no deben olvidarse a algunas líderes femeninas del siglo XX que ocuparon primeros puestos políticos en sus países participando en períodos claves de la historia, más allá del juicio político que pueda merecer esa contribución: Golda Maier en Israel; Indira Gandhi en la India; Eva Duarte de Perón en Argentina; Margaret Thatcher en Reino Unido; o Corazón Aquino en Filipinas, por ejemplo.

24. Véase Informe «El camino hacia una democracia sustantiva: la participación política de las mujeres en las Américas», Comisión Interamericana de Derechos Humanos, Organización de Estados Americanos, 20II. Recuperado el 2I de enero de 20I 5, desde http://www.oas.org/es/cidh/mujeres/docs/pdf/MUJERES\% 20PARTICIPACION\% 20POLITICA.pdf 
que cuentan con leyes de cuota que obligan a los partidos a incluir entre el $20 \%$ y el $40 \%$ de mujeres en las listas para elecciones legislativas (Argentina, Bolivia, Brasil, Costa Rica, Ecuador, Honduras, México, Panamá, Paraguay, Perú, República Dominicana y Venezuela) La paridad en tanto, muchas veces entendida como sinónimo de cuotas, busca más bien una extensión de la ciudadanía efectiva de las mujeres en todos los espacios, no sólo lo público, con una pretensión más ambiciosa y global que meramente cuantitativa.$^{25}$

$\mathrm{Al}$ año 2016 , Chile se encuentra recién ad portas de la entrada en funcionamiento de un nuevo sistema electoral que busca establecer mayor igualdad entre hombres y mujeres y que ha sido creado por la Ley No. 20.840. Si bien las mujeres votan desde 1949 en elecciones presidenciales y parlamentarias y cuentan con representación en la Cámara de Diputados desde I950 y en el Senado desde 1953, existe una infra representación en los cargos de elección popular, como fue mencionado en la introducción. Siendo en la actualidad más de la mitad del electorado nacional y a pesar del alza relativamente sostenida de los últimos años, la representación política de las mujeres en el poder legislativo nacional está muy por debajo del promedio mundial de 21,30\%, bajo el promedio de las Américas, del 24, I \% y bajo el promedio de todas las regiones del mundo (Informe Auditoría a la Democracia, 20I4).

En nuestro caso, además de las razones históricas y sociológicas que se recogen en este apartado más arriba, se ha señalado desde diversas institucionalidades internacionales que el tema encuentra como fuentes una serie de factores que podríamos calificar de más formales $-\mathrm{y}$ legales en algunos casos- como el tipo de sistema electoral (Informe Auditoría a la Democracia, 20I4), el funcionamiento cerrado y tradicional de los partidos políticos o la ausencia de medidas de acción afirmativa durante muchos años (Informe sobre los Derechos de las Mujeres en Chile, 2009), temas todos cuyo análisis en detalle exceden con mucho las pretensiones de este trabajo, pero que deben ser constatados.

A partir de este marco y teniendo en vista las cifras que muestra la realidad nacional, en este artículo se quiere mostrar la percepción de la importancia de la participación de las mujeres en política en Chile, identificada a partir del trabajo de análisis de las respuestas a las preguntas concretas de la encuesta relacionada, específicamente en las carreras pertenecientes a las Facultades de Educación, de Ciencias Sociales, y de Ciencias Jurídicas y Sociales, ello por la

25. ZÚÑIGA (20I3) p.I90 
formación que buscan entregar, que invita a suponer una mayor afinidad de quienes optan por ellas hacia los temas que ocupan estas páginas.

\section{Metodología}

El estudio efectuado ha sido de tipo transversal, ya que la medición se realizó en un solo instante; descriptiva, porque permitió caracterizar la muestra y sus subgrupos; comparativa, porque comparamos distintos grupos; y no experimental, porque no existe intervención de parte de los investigadores.

La población de interés fueron todos los estudiantes que ingresaron a primer año a la Universidad de Concepción, en su campus Concepción durante el año 20I3, de los cuales un total de I 249 fueron validados como casos para análisis. Por su parte, las variables de interés se han relacionado con los conocimientos y actitudes respecto a la democracia y la ciudadanía. El muestreo se realizó considerando como unidades de muestreo las distintas carreras cuyos estudiantes fueron escogidos al azar en forma proporcional. Específicamente en este trabajo se presenta la expresión de los resultados de los enunciados seleccionados, trabajados en 2 niveles de clasificación: resultado a nivel General Facultad Ciencias Sociales, Educación y Ciencias Jurídicas y Sociales y resultado por sexo, clasificado por Facultad Ciencias Sociales, Educación y Ciencias Jurídicas y Sociales.

En la Facultad de Ciencias Sociales, se ha encuestado a estudiantes de Trabajo Social, Antropología, Sociología, Periodismo y Psicología; en la de Ciencias Jurídicas y Sociales a estudiantes de Derecho y Ciencias Políticas y Administrativas; y en el caso de Educación a quienes ingresaron a estudiar las catorce carreras que ofrece.

El instrumento utilizado fue la encuesta de conceptualizaciones, actitudes y acciones utilizada en la prueba de la International Association for the Evaluation of Educational Achievement (IEA). La encuesta está constituida en base a 196 preguntas de selección múltiple dividas en 20 secciones, entre las que destacan pronunciamientos respecto a la democracia, los buenos ciudadanos, el Estado, la confianza en las instituciones, nuestro país, oportunidades, el sistema político, el currículo, uso de la fuerza militar, la acción política, efectividad de la acción política, sentimientos comunitarios, importancia de la toma de decisiones, la atención a los medios de comunicación, etc. De estas, se escogieron $\mathrm{I} 2$ áreas relevantes para los fines generales de la investigación y 8 para los fines concretos de este trabajo de exposición de resultados. 
El objetivo global de la prueba fue medir los conocimientos y actitudes de los estudiantes en relación a tres grandes ejes, democracia y ciudadanía; identidad nacional y relaciones internacionales; discriminación y tolerancia.

A partir de las respuestas de los alumnos a las preguntas seleccionadas se pretendió construir tres escalas de resultados cognitivos: conocimiento de contenidos, conocimiento de habilidades interpretativas y conocimiento cívico total. Para cada una de éstas se asignó un valor al promedio para estas escalas y otro a la desviación estándar, lo que sirvió especialmente para el análisis de otros de los aspectos consultados a los y las estudiantes.

También se calcularon varias escalas relativas al Compromiso Cívico y a las Actitudes y Otros Conceptos Cívicos de los estudiantes. La construcción de estas escalas consideró subconjuntos de ítemes de la prueba que apuntan a un mismo concepto o tema. Se asignó un valor al promedio para esas escalas y otro a la desviación estándar.

En cuanto al procesamiento de la información, el análisis estadístico realizado contempló una parte descriptiva, constituida por estimaciones de los porcentajes totales, para lo cual se consideraron ponderadores asociados a los tamaños relativos de los distintos grupos o áreas profesionales consideradas. Se completó esta parte descriptiva con los gráficos de comparaciones de porcentaje de respuestas favorables entre las distintas áreas profesionales y algunos gráficos de importancia estadística que ayudan a la interpretación de resultados.

El aspecto inferencial del análisis comprendió varios aspectos. El primero de ellos abordó la comparación de frecuencia de respuestas afirmativas entre las distintas áreas profesionales, utilizando la prueba Chi Cuadrado de homogeneidad de proporciones. El segundo aspecto del análisis inferencial se relaciona con la Construcción de Escalas de Conocimiento Cívico. Para este fin se realizaron dos procedimientos. El primero consistió en analizar la sección A de la encuesta, asociada a la Democracia, para la cual se extrajeron tres Factores mediante un análisis de Componentes Principales. Estos componentes principales fueron interpretados en término de cómo se relacionan con las preguntas y dimensiones de la encuesta. Además se estudió la influencia del área profesional sobre dichos Componentes Principales, mediante un análisis de varianza y, gráficamente, mediante los diagramas de Bi-Plot. El segundo procedimiento abordó toda la encuesta, la cual fue validada en términos de su consistencia interna, mediante el estadístico Alfa de Crombach. En base a este análisis se reconstruyeron las escalas de cada sección del instrumento con 
las preguntas que admitía dicha consistencia. El cálculo del estadístico utilizó ítemes transformados, es decir, respuestas orientadas en forma positiva, para no producir discrepancias entre preguntas para las cuales valores grandes implican respuesta correcta y otros para los cuales valores grandes implican respuesta incorrecta. Posteriormente se analizó la influencia del área profesional sobre cada escala, mediante Análisis de Varianza, y se obtuvieron gráficos que ilustran las comparaciones. El tercer aspecto del análisis inferencial consistió en la construcción de Clusters o agrupaciones de carreras, los cuales fueron ilustrados mediante un Dendograma, interpretando su sentido. Las pruebas de análisis de varianza fueron paramétricas o no paramétricas, según la naturaleza de los datos recolectados, conforme al cumplimiento de supuestos de normalidad, homocedasticidad e independencia.

Sobre estas bases metodológicas, la investigación se ejecutó en tres etapas, entre los años 20I3, 20I 4 y 20I 5 . La primera comprendió una profundización del marco teórico a través de una revisión profunda de la literatura especializada y la configuración del marco muestral. Además la firma de los «Protocolos para la Obtención del Consentimiento Informado» y el diseño de los protocolos correspondientes, concluyendo con la adquisición de los materiales que sirvieron para la realización de la investigación. La segunda etapa se inició con el levantamiento de la información y su transcripción en formato digital para efectuar los análisis estadísticos correspondientes. En esta etapa se realizó un preanálisis de la información obtenida, obteniendo un primer informe de avance, discutido entre los investigadores. La tercera etapa tuvo lugar durante los años finales del proyecto y tuvo como propósito realizar el análisis final de la información estadística, analizándola a la luz de la literatura especializada, a partir de una re-clasificación de la información. En la ejecución de esta tercera etapa se inserta el análisis específico cuyos resultados se exponen a continuación.

\section{Exposicion y analisis de los resultados obtenidos}

A la luz de los movimientos estudiantiles de los últimos años, no hay duda de que los y las jóvenes universitarios tienen una opinión sobre lo público, y especialmente en relación al sistema educacional del cual forman parte. A diferencia de las generaciones anteriores quienes consideraban el voto tanto un deber como derecho ciudadano, las generaciones nacidas en democracia a menudo cuestiona la representatividad de la actual democracia (Luna, 20 I I). 
En comparación con sus padres, los y las estudiantes universitarios de hoy ya no están ligados tan fuertemente a su origen social, ya que las mejores oportunidades educativas y las nuevas opciones en el mercado laboral hacen que la movilidad social sea posible. Los elementos que estructuran el comportamiento político son cada vez más difusos, especialmente entre los electores más jóvenes. A diferencia de electores mayores, quienes se identifican más con los partidos políticos y posiciones partidistas, los jóvenes consideran los elementos personales de los candidatos y las cuestiones políticas puntuales (Toro, 2007).

De hecho, Luna plantea que los campos identitarios asociados a los dos grandes pactos políticos electorales, los cuales están basados en la historia reciente, no logran ni encantar ni movilizar electoralmente a las nuevas generaciones que se integran a la ciudadanía (Luna, 20II). Además, en comparación con votantes mayores, los jóvenes chilenos (menores de 35 años) parecen valorar menos su participación en los procesos de decisión democrática. Tercero, los jóvenes que no se estaban inscritos antes de la reforma, desconfían y se encuentran fuertemente alejados del sistema político.

Algunos autores, como Delli con su estudio en Estados Unidos, plantean que la desafección se relaciona con la falta de identidad ciudadana, el bajo conocimiento respecto a los procesos políticos y una falta de confianza en sus conciudadanos (Delli, 2000).

No obstante, otros autores como Martínez, Silva y Hernández (2010), plantean que la desafección política al sistema formal no siempre significa un rechazo a la participación en actividades no institucionalizadas como la protesta pública. Dicha desconfianza en la política partidista combinada con el aumento de la protesta pública se observan en casi todos los países (Norris, I999).

Por lo anterior y teniendo en vista la realidad estadística de las mujeres y su participación política en Chile, conviene preguntarse y analizar los conocimientos y actitudes que poseen estudiantes que ingresan a primer año en la Universidad de Concepción respecto a la participación específica de las mujeres y sus oportunidades. En forma complementaria y previa además, sobre el rol que tiene esta participación en la democracia, desde los deberes del estado y la confianza en las instituciones. 


\subsection{Democracia, estado y confianza en las instituciones}

El gráfico I expone los resultados que arroja entre los y las encuestados la pregunta sobre si es bueno para la democracia el que los partidos políticos tengan normas que apoyan a las mujeres para que lleguen a ser dirigentes políticos. El resultado general a nivel de las tres facultades que nos interesan indica que la percepción mayoritaria es que esto es bueno o muy bueno para la democracia (porcentajes por sobre el $40 \%$ en los tres casos) y que sólo un porcentaje menor al $5 \%$ lo estima malo o muy malo. Al desagregar estos datos por sexo (gráfico 2), se puede constatar que si bien se mantiene la proporción mayoritaria hacia considerarla bueno o muy bueno para la democracia, sólo en la Facultad de Ciencias Jurídicas y Sociales el porcentaje de mujeres es superior en ambas clases de respuesta. En las Facultades de Ciencias Sociales y de Educación, es mayor el porcentaje de hombres que se pronuncia en este sentido.

Lo anterior puede deberse a que la Facultad de Ciencias Jurídicas y Sociales incorpora la carrera de Derecho en que tradicionalmente existe una formación mayor en tema de igualdad de oportunidades entre hombres y mujeres, desde el punto de vista de la enseñanza de la norma legal, al menos en el currículum

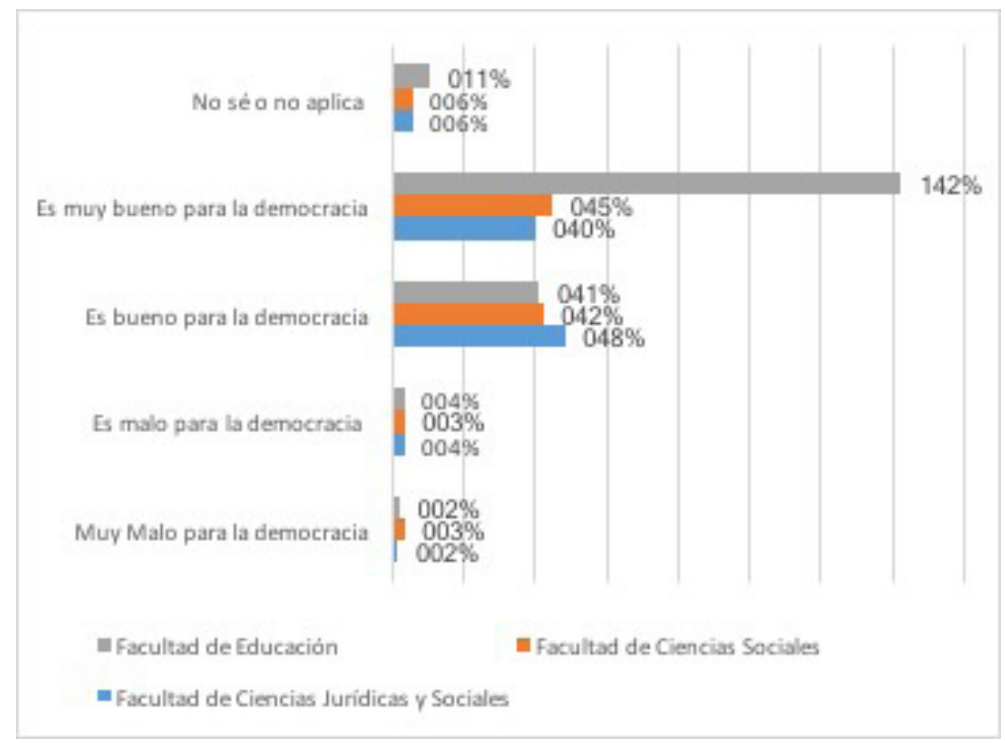

Gráfico 1. ¿Qué es bueno y qué es malo para la democracia? «Cuando los partidos políticos tienen normas que apoyan a las mujeres para que lleguen a ser dirigentes políticos.» Resultado a nivel General Facultad Ciencias Sociales, Educación y Ciencias Jurídicas y Sociales. Fuente: Elaboración propia. 


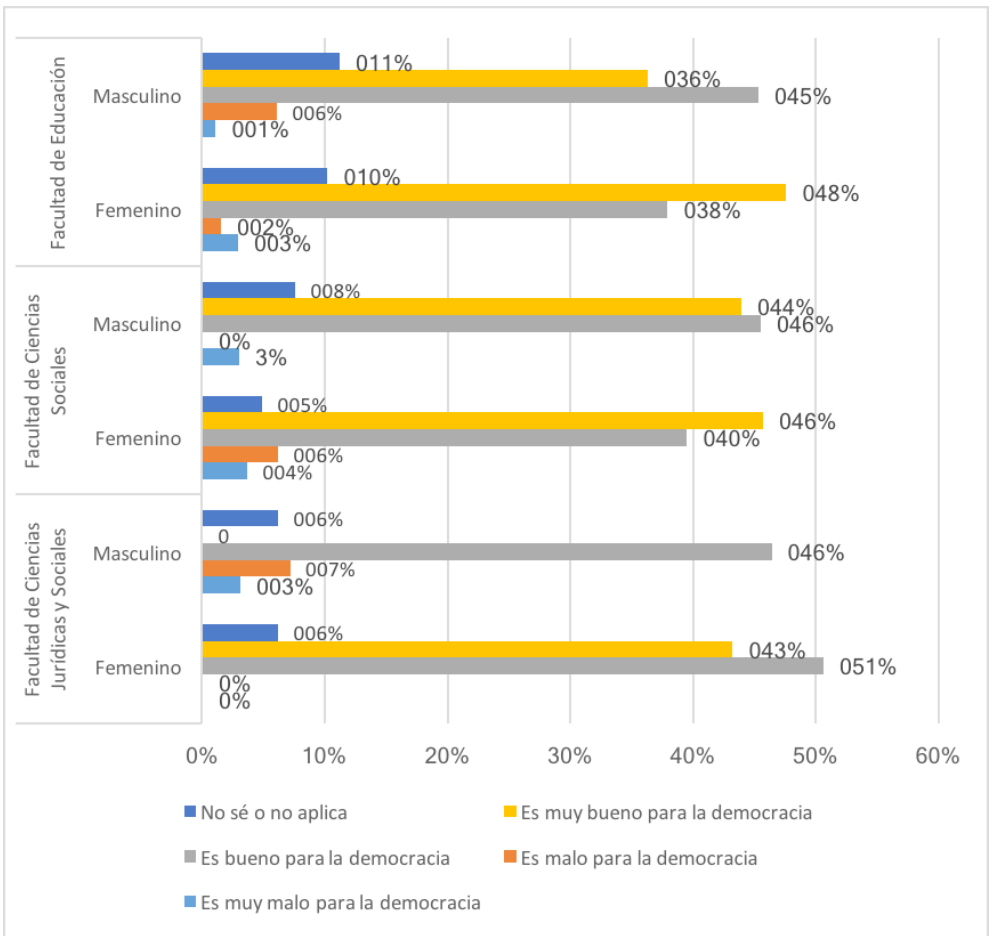

Gráfico 2. ¿Qué es bueno y qué es malo para la democracia? «Cuando los partidos políticos tienen normas que apoyan a las mujeres para que lleguen a ser dirigentes políticos.» Resultado por sexo clasificado por Facultad Ciencias Sociales, Educación y Ciencias Jurídicas y Sociales. Fuente: Elaboración propia.

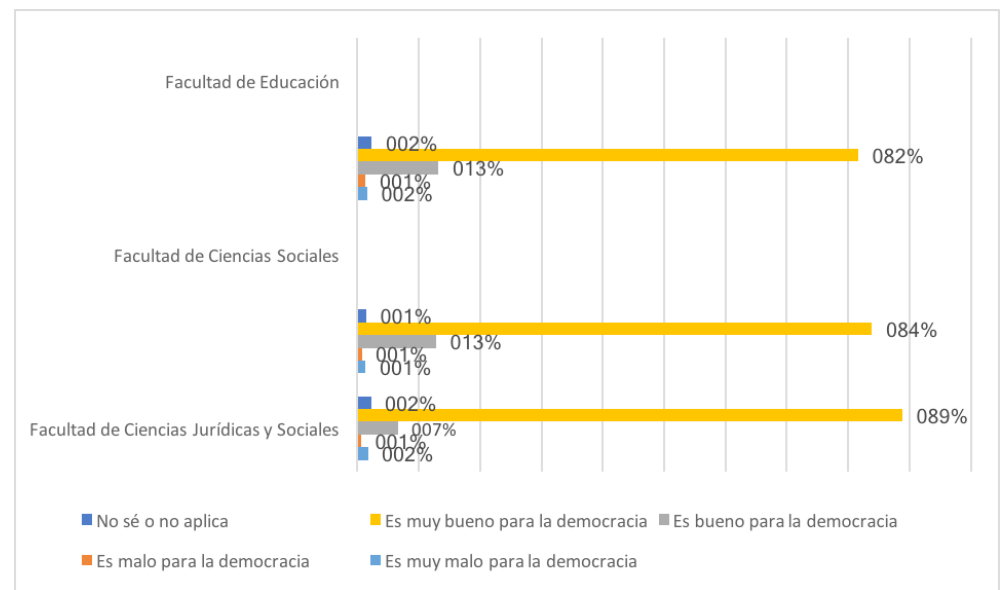

Gráfico 3. ¿Qué es bueno y qué es malo para la democracia?

«Cuando los ciudadanos tienen el derecho a elegir libremente a sus dirigentes políticos». Resultado a nivel General Facultad Ciencias Sociales, Educación y Ciencias Jurídicas y Sociales. Fuente: Elaboración propia. 


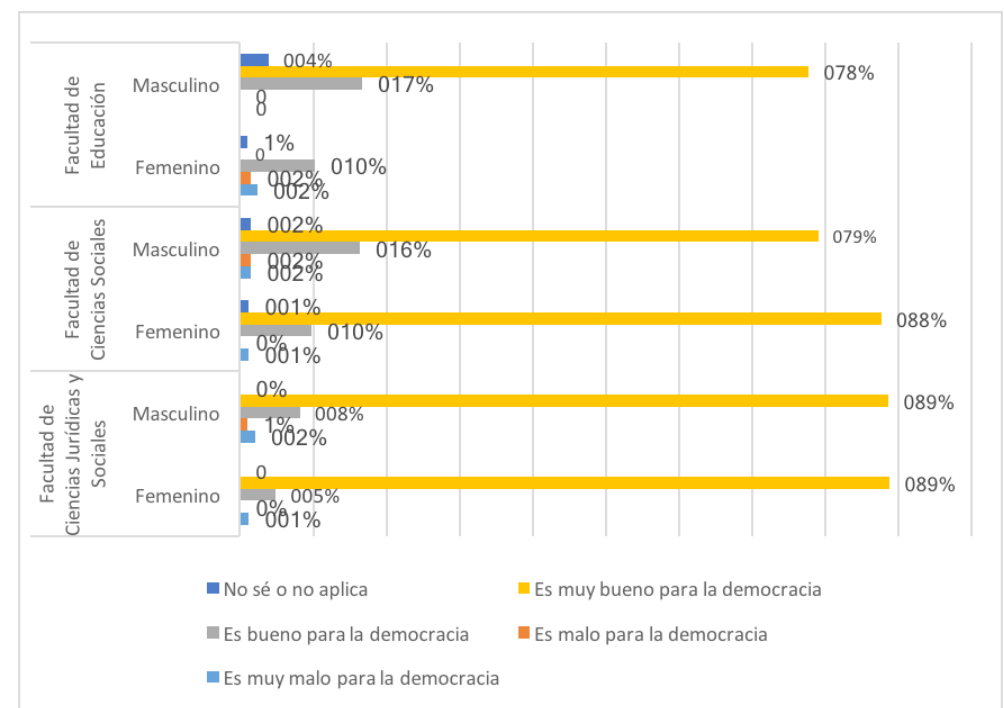

Gráfico 4. ¿Qué es bueno y qué es malo para la democracia? "Cuando los ciudadanos tienen el derecho a elegir libremente a sus dirigentes políticos». Resultado por sexo clasificado por Facultad Ciencias Sociales, Educación y Ciencias Jurídicas y Sociales. Fuente: Elaboración propia.

formal de asignaturas como Instituciones Políticas, Derechos Fundamentales, Derecho Constitucional y Derecho Internacional.

En forma adicional, se consultó si era bueno o no para la democracia cuando los ciudadanos tienen el derecho a elegir libremente a sus dirigentes políticos. En este punto el porcentaje «es muy bueno para la democracia» en las tres facultades supera el $80 \%$, mientras que «es muy malo para la democracia» o «es malo para la democracia», no alcanza al $2 \%$ en todas (gráfico 3 ).

Si comparamos con los resultados que arrojan la pregunta anterior vemos que si bien hay una buena valoración respecto a que es bueno para la democracia el que los partidos políticos tengan normas que apoyan a las mujeres para que lleguen a ser dirigentes políticos, tiene una mucho más alta valoración para la democracia la libertad de elegir a los dirigentes políticos, valoración que es bastante similar entre hombres y mujeres si desagregamos el dato (gráfico 4).

Estos resultados se podrían relacionar con la percepción que tienen de los partidos políticos y la confianza en las instituciones.

En efecto, frente a la pregunta sobre con qué frecuencia puedes confiar en los partidos políticos en cuanto institución, las respuestas negativas son sorprendentemente altas y la respuesta «siempre» no alcanza al I \% en ninguna de las facultades consultadas, siendo la opción «nunca» aquella que obtiene la 


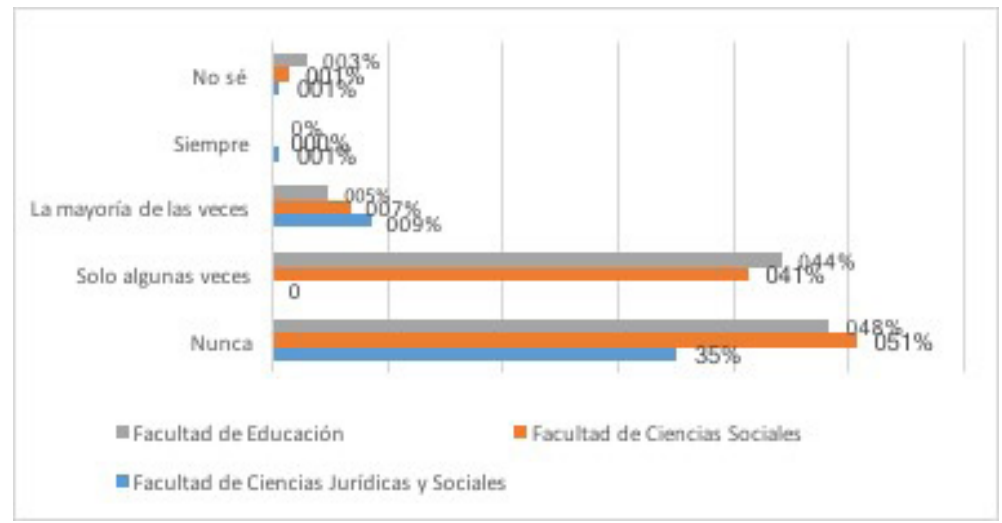

Gráfico 5. ¿Con qué frecuencia puedes confiar en cada una de las siguientes instituciones? «Los partidos políticos». Resultado a nivel General Facultad Ciencias Sociales, Educación y Ciencias Jurídicas y Sociales. Fuente: Elaboración propia.

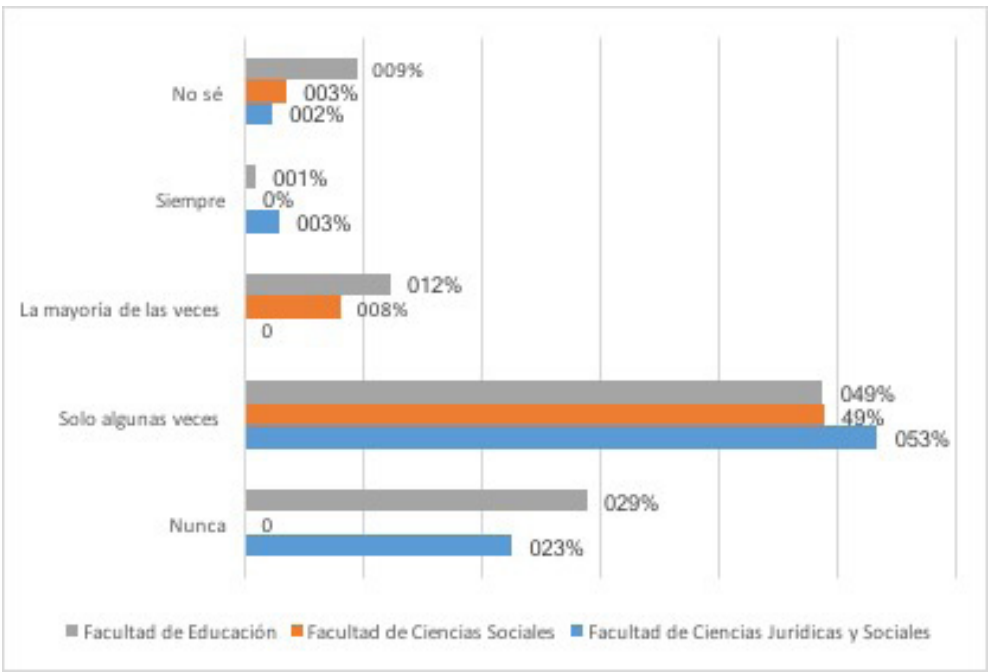

Gráfico 6. ¿Con qué frecuencia puedes confiar en cada una de las siguientes instituciones? «El Congreso» Resultado a nivel General Facultad Ciencias Sociales, Educación y Ciencias Jurídicas y Sociales. Fuente: Elaboración propia.

mayor adhesión en el caso de las facultades de Ciencias Sociales y de Educación. En el área de ciencias jurídicas y sociales la negativa se atenúa un poco al ser mayor el porcentaje que opta por la respuesta «sólo algunas veces» (gráfico 5). Efectuada la misma pregunta pero ahora en relación a la confianza en el Congreso Nacional, la tendencia es muy similar

Por su parte, al consultar sobre la responsabilidad que le cabe al Estado en 


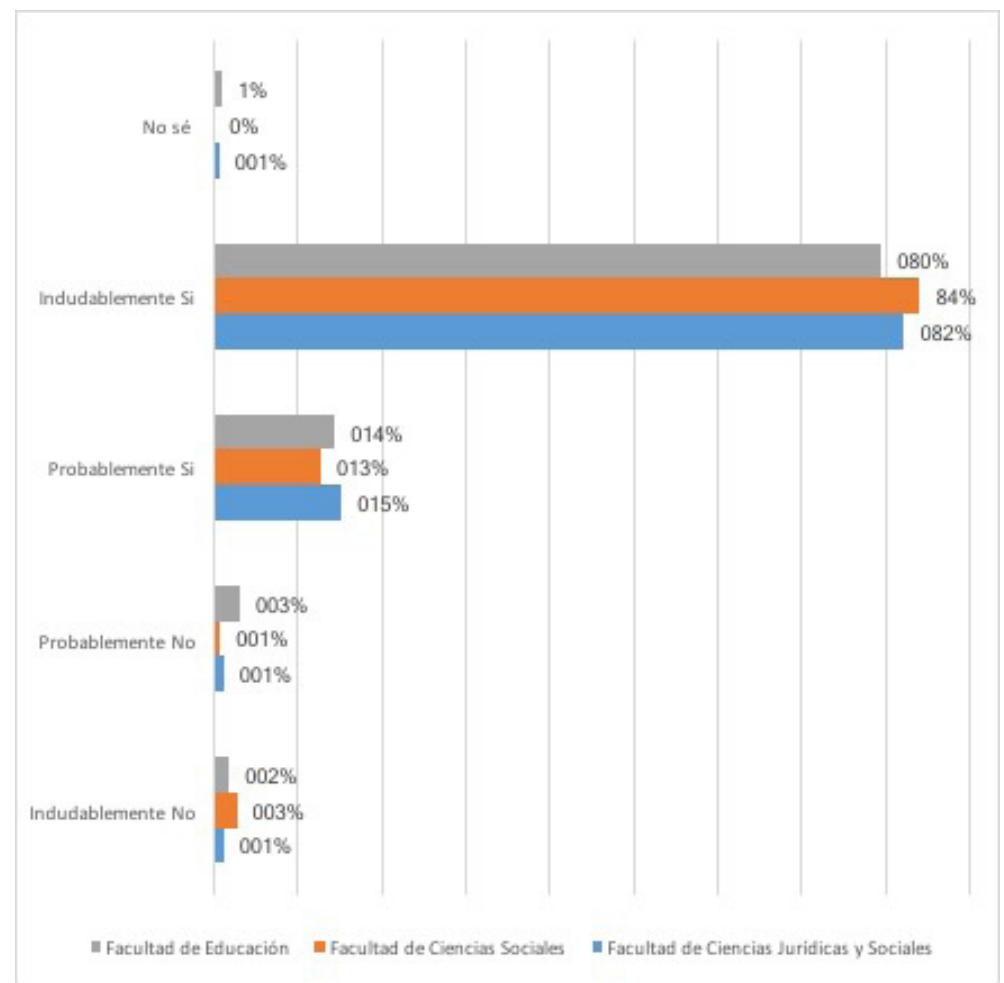

Gráfico 7. ¿Qué responsabilidades debería tener el Estado? «Asegurar igualdad de oportunidades políticas para hombres y mujeres.» Resultado a nivel General Facultad Ciencias Sociales, Educación y Ciencias Jurídicas y Sociales. Fuente: Elaboración propia.

este tema, la confianza parece ser bastante mayor que en los partidos políticos y en el Congreso Nacional, al estimar que es parte «indudablemente» de sus deberes asegurar igualdad de oportunidades políticas para hombres y mujeres en más de un $80 \%$ (gráfico 7 ).

Es interesante la manifestación de desconfianza en los partidos políticos y en el Congreso Nacional. Ello supondría que los jóvenes que ingresan al mundo estudiantil universitario no vinculan necesariamente la ciudadanía o el ejercicio de la democracia con la participación en un partido político.

\subsection{Participacion politica de mujeres y oportunidades}

La tabla I, muestra los resultados obtenidos al consultar la percepción respecto de algunas afirmaciones acerca de las oportunidades que los integrantes de ciertos grupos «DEBERÍAN TENER» en Chile. 
Tabla 1. Oportunidades. Postura mayormente muy positiva y en algunos casos variable, hacia los conceptos vinculados a las oportunidades. Fuente: Elaboración propia.

\begin{tabular}{|lccccc|}
\hline Ítem & $\mathbf{1}$ & $\mathbf{2}$ & $\mathbf{3}$ & $\mathbf{4}$ & No sabe \\
\hline E.1 & $2 \%$ & $1 \%$ & $26 \%$ & $70 \%$ & $2 \%$ \\
\hline E.2 & $1 \%$ & $1 \%$ & $16 \%$ & $81 \%$ & $1 \%$ \\
\hline E 3 INVERTIDA & $8 \%$ & $16 \%$ & $45 \%$ & $22 \%$ & $10 \%$ \\
\hline E.4 & $1 \%$ & $2 \%$ & $19 \%$ & $77 \%$ & $1 \%$ \\
\hline E.5 & $1 \%$ & $1 \%$ & $19 \%$ & $78 \%$ & $1 \%$ \\
\hline E.6 INVERTIDA & $4 \%$ & $4 \%$ & $17 \%$ & $73 \%$ & $2 \%$ \\
\hline E.7 INVERTIDA & $9 \%$ & $14 \%$ & $38 \%$ & $20 \%$ & $20 \%$ \\
\hline E.8 & $1 \%$ & $1 \%$ & $15 \%$ & $81 \%$ & $1 \%$ \\
\hline E.9 INVERTIDA & $3 \%$ & $6 \%$ & $26 \%$ & $62 \%$ & $3 \%$ \\
\hline E.10 INVERTIDA & $9 \%$ & $17 \%$ & $38 \%$ & $21 \%$ & $15 \%$ \\
\hline E.11 & $1 \%$ & $2 \%$ & $14 \%$ & $82 \%$ & $1 \%$ \\
\hline E.12 & $3 \%$ & $11 \%$ & $42 \%$ & $33 \%$ & $11 \%$ \\
\hline E.13 INVERTIDA & $3 \%$ & $6 \%$ & $21 \%$ & $64 \%$ & $6 \%$ \\
\hline
\end{tabular}

Frente a cada interrogante se les dio la oportunidad de responder - en base a su manera o forma de pensar - entre estar «Muy en desacuerdo»; «En desacuerdo", "De acuerdo»; «Muy de acuerdo»; o manifestarse indicando no saber.

A la consulta sobre si las mujeres deberían ser candidatas al Congreso y participar en el gobierno al igual que los hombres (E.I) el $70 \%$ se muestra muy de acuerdo, mientras que el $26 \%$ dice estar de acuerdo, quedando las opciones negativas con menos del $5 \%$, lo que reflejaría la asunción de un discurso formal positivo, percepción que se mantiene bastante parecida cuando se consulta si las mujeres deberían tener los mismos derechos que los hombres en todos los aspectos (E.4) Ello significaría que están cada vez más conscientes de sus derechos y de la importancia de reconocer el derecho de quienes históricamente han ido quedando más excluidos, como las mujeres.

No obstante, frente a la interrogante de si las mujeres deberían mantenerse al margen de la política (E.6) los resultados muestran una inversión de la percepción al constatar que más del 70\% está muy de acuerdo con esa afirmación y un $17 \%$ dice estar de acuerdo, lo que mostraría que si bien hay un recono- 
cimiento a que las mujeres deben poder participar políticamente en igualdad de condiciones, a la hora de generar oportunidades reales, el estudiantado consultado prefiere que queden al margen de esos procesos.

Esta aseveración encuentra fundamento al contrastar los resultados de dos preguntas inversamente relacionadas. En la interrogante si deberían hombres y mujeres que desempeñan el mismo trabajo recibir la misma remuneración (E.II), el $82 \%$ se muestra muy de acuerdo y menos de un $5 \%$ favorece las respuestas negativas. Sin embargo, al interrogar acerca de si los hombres están mejor calificados que las mujeres para ser dirigentes políticos (E.I3) el porcentaje muy de acuerdo baja al $64 \%$.

\section{Reflexiones finales}

Los resultados de la investigación que han sido expuestos en este trabajo demuestran que - como se anunció en la introducción - pese a contar por segunda vez con una mujer como Jefa de Estado y Jefa de Gobierno, siendo las mujeres en lo global la mitad de la población chilena y existiendo desarrollos teóricos, normativo e institucionales que llaman a favorecer la histórica exclusión de las mujeres en diversos ámbitos, como es el político, faltan espacios en que ser protagonistas verdaderamente.

En efecto, al mirar la realidad es posible comprobar que pese a las normas, los discursos sociales y las políticas públicas, las mujeres siguen sufriendo diversas manifestaciones de desigualdad y el mundo de la política es un buen ejemplo de ello. El empoderamiento de las mujeres está lejos de llegar en plenitud.

La lectura integrada de las respuestas de las y los encuestados en esta investigación permite aseverar que no es fácil el camino que queda por andar y que desde el discurso formal a la realidad hay un amplio trecho.

Los y las estudiantes en el inicio de su vida académica universitaria estarían conscientes de sus derechos y de la importancia de reconocerlos a las mujeres, desde que históricamente han estado excluidas del ejercicio de poder político en las distintas facetas de la participación. Asimismo, valoran positivamente la consagración formal de su participación política, consideran un deber del Estado garantizar la igualdad de oportunidades entre mujeres y hombres y muestran una gran desconfianza en los partidos políticos y en el Congreso Nacional, lo que supondría que no vinculan necesariamente la ciudadanía o el ejercicio de la democracia con la participación en un partido político. 
Sin embargo, a la hora de las oportunidades, la confianza tiende a ser mayor en los hombres para depositar responsabilidades de carácter político, lo que introduce la necesidad de avanzar desde la formalidad del buen discurso sobre igualdad y derechos a la acción práctica en política teniendo en este punto la universidad una gran responsabilidad que asumir en cuanto formadora de los y las ciudadanas del futuro.

Si bien se vienen tiempos más auspiciosos desde un punto de vista formal con la introducción de cuotas en el sistema electoral chileno y los importantes cargos públicos que mujeres han ganado en elecciones democráticas en el país, los avances que se vayan logrando deben asegurar realmente mayores oportunidades de participación de las mujeres y lograr mejorar la percepción de una parte de la ciudadanía - estos jóvenes universitarios, por ejemplo - que parece que todavía cree que los hombres serán mejores líderes o articulares de lo público político que las mujeres, imagen que puede tener diversas explicaciones.

Si se toma como referencia el marco teórico descrito en la primera parte de este trabajo se podría especular que una de esas explicaciones puede estar radicada en aquella idea de que estamos inmersos en una cultura "humana» que ha tenido y tiene una fuerte carga androcéntrica en la construcción e implementación de la mayoría de sus instituciones, desde las políticas y sociales a las jurídicas, y en la cual se han estructurado espacios y lugares sólo para hombres (lo público) y espacios y lugares sólo para mujeres (lo privado).

Reafirma lo anterior la propia encuesta de Corporación Humanas que antes hemos referido en este trabajo ya que, ante la sección «Roles de género» $y$ la pregunta de cuán de acuerdo o no están las encuestadas con la afirmación «La mujeres deben trabajar siempre que no descuiden a su familia» el 90,9\% está de acuerdo, a pesar de que la valoración en general al trabajo de mujeres fuera del hogar es muy alta ${ }^{26}$. Por su parte, ante la pregunta «Frente a la política, ¿usted se considera una persona muy interesada, interesada, desinteresada o muy desinteresada?» un $42,7 \%$ se muestra desinteresada, un $23,3 \%$ muy desinteresada, un $27,6 \%$ interesada y sólo un $6,0 \%$ muy interesada, lo que es consecuente con la respuesta ante la implicancia de la política en sus

26. En la misma sección de la Encuesta «Roles de género» y la pregunta de cuán de acuerdo o no están las encuestadas con la afirmación «El trabajo remunerado les da a las mujeres independencia económica y realización personal» el 95,4\% está de acuerdo. 
interacciones sociales: $59,7 \%$ contesta que nunca es tema en el marco de estas interacciones.

En 20 Io ya lo dijo el Informe del PNUD sobre Chile - y poco hemos avanzando en esto - "es posible afirmar que las mujeres no están plenamente incorporadas al ejercicio del poder en las organizaciones formales tradicionales. No se trata sólo de la distribución porcentual de los cargos en los distintos ámbitos de la toma de decisiones, sino en la 'manera de hacer las cosas' en esas esferas. Los lenguajes, los temas, los horarios, la forma de sociabilidades y el uso de los espacios, las estrategias de alianza, el manejo de los conflictos y los estilos de liderazgo, todos ellos reproducen de manera imperceptible el predominio masculino" (Informe Desarrollo Humano en Chile, 20го), a lo que podemos agregar el valor simbólico en sociedades como la nuestra sobre los cuestionamientos implícitos soterrados sobre la real capacidad de decidir de las mujeres y sobre cómo vamos en verdad a compatibilizar el rol político público con la familia, sin que dichos cuestionamientos -instalados en el imaginario social inconsciente - se hagan presente cuando de hombres hablamos.

Por ahí parece entonces que puede ir una explicación a esa disociación que encontramos entre el discurso formal y las respuestas de nuestros y nuestras estudiantes.

Frente a ello, la educación se tendría que levantar como un articulador de un modelo nuevo en este ámbito de las demandas de las mujeres, que permita pasar a la realidad esa asunción formal que parece instalada en el colectivo social sobre la menor participación de las mujeres en política y la necesidad de promover más y mejores oportunidades.

Pensamos no sólo en la educación formal que se pueda brindar a través del currículum escolar o universitario, sino también de aquella que se puede hacer informalmente por otros medios. Por ejemplo, con la mayor promoción de la participación de mujeres en otros ámbitos de la política, tales como las asociaciones voluntarias o los centros estudiantiles que podríamos llamar «baja política» y que permitan transitar así a esa inserción real de las mujeres en la «alta política»: la de los votos y las decisiones por el poder estatal o local. $\mathrm{O}$ contribuyendo desde la vida personal a generar una nueva conciencia, que elimine aquellos estereotipos o concepciones sobre lo que realmente podemos hacer o no hacer las mujeres. 


\section{Referencias}

Beltrán, Elena y Maqueira, Virginia (Eds.) (2008): Álvarez, Silvina y SánCHEz, Cristina: Feminismos: Debates teóricos contemporáneos (Madrid, Alianza Editorial S.A.).

Delli Carpini, Michael (2000): «Gen.Com: Youth, Civic Engagement and the New Information Environment» en Political Communication, No.I7, pp. 34I-349.

Fries, Loren y Matus, Verónica (I999): El Derecho. Trama y conjura patriarcal (Santiago, LOM Ediciones).

Gauché, Ximena (20I I): Sexualidad Diversa y Discriminación. Una mirada desde el Derecho Internacional de los Derechos Humanos (Saarbrücken, Editorial Académica Española).

Programa de Naciones Unidas para el Desarrollo (20I4): Informe Auditoría a la Democracia. Más y mejor democracia para un Chile inclusivo. (Santiago, Programa de Naciones Unidas para el Desarrollo)

Programa de Naciones Unidas para el Desarrollo (20io): Informe Desarrollo Humano en Chile. Género: los desafíos de la igualdad. (Santiago, Programa de Naciones Unidas para el Desarrollo)

Comisión Interamericana de Derechos Humanos (2009): InFOrme sobre los Derechos de las Mujeres en Chile. La igualdad en la familia, el trabajo y la política (Washington D.C., Organización de Estados Americanos). Fries, Lorena y Matus, Verónica (I999): El derecho. Trama y conjura patriarcal (Santiago, Lom ediciones).

Lamas, Marta (2013): Cuerpo, sexo y política. Colección Debate Feminista (México D.F., Editorial Océano).

LunA, Juan Pablo (20I I): «Jóvenes, inscripción automática y voto voluntario: ¿El tipo de reforma que debemos evitar?» En Temas de Agenda Pública, Año VI, $\mathrm{N}^{\circ} 46$, septiembre de $20 \mathrm{II}$, pp. I-I 2.

Macionis, Jhon y Plummer, Ken (2007), Sociología, $3^{a}$ edición (Madrid, Pearson Prentice Hall).

Martínez, M. Loreto, Silva Carmen y Hernández, Ana (20io): ¿En qué Ciudadanía Creen los Jóvenes? Creencias, Aspiraciones de Ciudadanía y Motivaciones para la Participación Sociopolítica», en PSYKHE, Vol. I9, $\mathrm{n}^{\circ} 2$, pp. 25-37.

Norris, Pippa (ed.) (I999): Critical Citizens (Oxford, Oxford University Press). 
Pasquino, Gianfranco (I994): Manual de ciencia politica (Madrid, Alianza). Pharr, Susan y Putnam, Robert (2000): Disaffected democracies: What's wrong with the trilateral democracies (Princeton, Princeton University Press).

Renau, María Dolors (2008): Ciudadanas y Políticas (Barcelona, Fundación Rafael Campalans).

Uña, Octavio y Hernández, Alfredo (2004): Diccionario de sociología (Madrid, Editorial ESIC).

Toro, Sergio (2007): «Participación Electoral de los Jóvenes en Chile: Factores de Incidencia en la Inscripción Juvenil». En Fontaine, Arturo; LARroulet, Cristián; Viera-Gallo, José Antonio y Walker, Ignacio (Eds.) (2007): Modernización del Régimen electoral chileno (Santiago, Programa de Naciones Unidas para el Desarrollo), pp. IоI-I 22.

ZuÑIga, Yanira (2013). "La igualdad de género en el sistema regional americano", en "Problemáticas Actuales del Derecho Constitucional». Actas de las IX Jornadas Constitucionales realizadas en 2013 en el Centro de Estudios Constitucionales y Administrativos de la Universidad Mayor. (Temuco, Editorial Universidad Mayor), pp. 3 I-42.

ZuÑIGA, Yanira (20I3): "Mujeres, ciudadanía y participación política», en Lacrampette, Nicole (Ed.) (2013): Derechos Humanos y Mujeres: Teoría y Práctica (Santiago, Centro de Derechos Humanos de la Universidad de Chile) pp. I 8 I-2 Io. 
